\title{
Securing communications over ATM networks
}

\author{
M. LAURENT - O. PAUL - P. ROLIN \\ Télécom Bretagne \\ rue de la châtaigneraie - BP 78 - 35512 CESSON Cedex - France \\ Tel: (33 2) 0299127040 \\ Fax: (33 2) 0299127019 \\ Email: \{mlaurent, rolin\}@ rennes.enst-bretagne.fr
}

\begin{abstract}
This paper presents a survey of existing solutions aiming to secure communications over ATM networks. Different solutions are analyzed and compared. Details are given about the security services offered, their placement within the ATM Protocol Reference Model, the mechanisms to negotiate security services and the techniques to provide synchronization and dynamic key change during user data exchange.

Additionally, this paper proposes a new ATM security solution - the Solution For Frequent Communications (SFFC) - suitable to protect communications between ATM stations or companies that need to frequently communicate. An implementation outline is also presented.
\end{abstract}

\section{Keywords}

B-ISDN, ATM, security network, security services.

\section{INTRODUCTION}

To support near future multimedia services, each requiring specific needs in terms of Quality of Service (QoS), ITU-T defined the Broadband Integrated Services Digital Network (B-ISDN) and adopted the Asynchronous Transfer Mode (ATM) as the technology to implement B-ISDN. ATM is still under development and one issue is that ATM facilities emerge although some ATM specifications/standards are still unavailable. About security issues, current ATM facilities (e.g. ATM switches) have many restrictions which are not favourable to simple and reliable security services introduction. That is why, it is time to secure ATM since sooner security specifications will be defined, simpler and more reliable the chosen security solution will be and wider the security services will be implemented within ATM facilities. 
In the literature many works introduce security services (in the sense of (UIT-T X.800, 1991)) into the ATM Protocol Reference Model described in section 2. Section 3 outlines and compares those schemes. Also a new scheme to make communications over ATM networks secure is proposed in section 4 and implementation details are given in section 5. Tables 2 and 3 resume those schemes' features and section 6 presents conclusions.

\section{THE ATM PROTOCOL REFERENCE MODEL}

As depicted in Figure 1, the ATM reference model (De Prycker, 1991), (CCITT I.321, 1991) includes three planes:

- the user plane to exchange user data,

- the control plane to monitor signaling information,

- the management plane to maintain the network operational.

User and control planes communicate over the ATM network through reserved virtual channels. The management plane may use the same

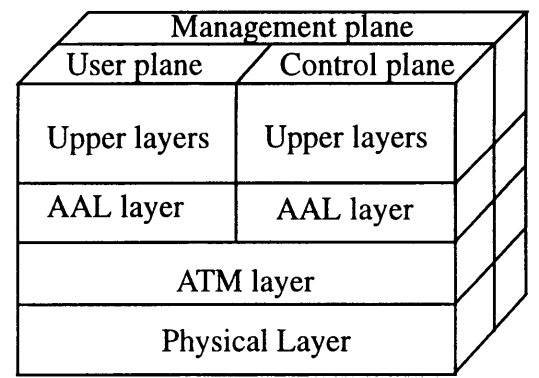

Figure 1 The ATM protocol reference. channel as the user plane to exchange Operation And Maintenance (OAM) cells. All information is exchanged over the network in the form of 53-byte ATM cells.

The ATM reference model includes three lower layers, a physical layer mainly responsible for information transportation, an ATM layer mainly in charge of multiplexing and switching functions and an ATM Adaptation Layer (AAL) whose main function is to adapt services needs to ATM streams by performing segmentation into (/reassembly of) cells for instance.

\section{SURVEY}

Solutions to secure ATM communications are discussed in (Stevenson, 1995), (Deng, 1995), (Chuang, 1996) and (ATM Forum, 1996). As securing an ATM communication is based on firstly negotiating a security context and secondly applying negotiated security services on user data exchanges, these solutions' presentation is divided into two corresponding subsections. Along the sections, notations of table 1 are used.

Table 1 : Notations

\begin{tabular}{|l|l|}
\hline A & calling entity \\
\hline B & called entity \\
\hline KAB & $\begin{array}{l}\text { session key generated by A to protect flow from A } \\
\text { to } B\end{array}$ \\
\hline KsA $\{M\}$ & encrypted M with A's private key \\
\hline KpA $\{M\}$ & encrypted $M$ with A's public key \\
\hline$T_{A}$ & current timestamp generated by $A$ \\
\hline$N_{A}$ & nonce generated by A \\
\hline CA & certification authority entity \\
\hline Cert_A & A's public key certificate generated by CA \\
\hline
\end{tabular}




\begin{tabular}{|c|c|}
\hline Security IE Identifier 1 byte & \multirow{13}{*}{$\begin{array}{l}\text { The IE's fields include (for details, refer to (ATM Forum,1996)): } \\
\text { - Authentication IE Identifier identifies the authentication IE } \\
\text { within signaling messages } \\
\text { - Version denotes the version to which the IE is compliant } \\
\text { - E/E indicates end-to-end ( } E / E=1 \text { ) or hop-by-hop }(E / E=0) \\
\text { authentication } \\
\text { - Signature algorithm identifies the code of the algorithm used } \\
\text { for generating the signature } \\
\text { - Sequence number/timestamp allow each authentication IE to be } \\
\text { unique } \\
\text { - IE list length and IE list contain respectively the number of IEs } \\
\text { included into the IE list field and the list of IE's identifiers that } \\
\text { specifies the IEs over which the signature is computed and the } \\
\text { ordering of IEs during generation } \\
\text { - Algorithm-specific information includes parameters to be used } \\
\text { for signature generation } \\
\text { - Signature contains the digital signature }\end{array}$} \\
\hline IE $\mathrm{i}$ & \\
\hline Length 2 by & \\
\hline Version & \\
\hline Signature Algorithm & \\
\hline Sequence number & \\
\hline Timestamp ( 4 bytes) & \\
\hline IE list lenth & \\
\hline IE list & \\
\hline Encrypted IEs & \\
\hline Reserved & \\
\hline orithm-specific inforn & \\
\hline & \\
\hline
\end{tabular}

Figure 2 Authentication IE format of the ATM Forum's solution.

\subsection{Security services negotiation}

ATM security solutions differ in that security services negotiation is done :

- during connection set up within control operations (ATM Forum, 1996), (Deng, 1995), (Chuang, 1996)

- along the connection within management operations (ATM Forum, 1996)

- over an auxiliary channel dedicated to security operations (Stevenson, 1995), (ATM Forum, 1996)

Note that ATM Forum's solution uses all these schemes to exchange security information.

\subsubsection{Negotiation through signaling information}

This scheme consists in inserting security information (session keys, security mechanisms, authenticator) into signaling messages such as SET UP* and CONNECT ${ }^{\dagger}$ within one or more Information Elements ${ }^{\ddagger}$ (IEs).

\subsubsection{The ATM Forum's solution}

The ATM Forum limits signaling support to the authentication and access control services. Other security services are realized by other means presented in sections 3.1.2.1 and 3.1.3.2.

The ATM Forum defines a new IE whose format is given in Figure 2 and which allows to authenticate signaling messages' source. Authentication may be done end-to-end (between two end-entities) or hop-by-hop (between switches and end-entities) in connection set up messages (SET UP and CONNECT) and other signaling messages such as connection release.

Additionally to the authentication service, the IE ensures integrity of part or all the signaling message and replay detection with the Sequence number and Timestamp fields.

One access control specific IE is also defined to specify the sensitivity level of user data that need to be exchanged over the connection so that, for instance, the path selected over the network uses only links with appropriate sensitivity level.

*. SET UP is sent by A to initiate a call establishment.

$\dagger$. CONNECT is sent by B in response to SET UP messages to indicate call acceptance.

‡. An Information Element is a structure within signaling messages that include one type of information useful for control operations. E.g. A and B's addresses are included within SET UP as calling and called entity number IEs. 


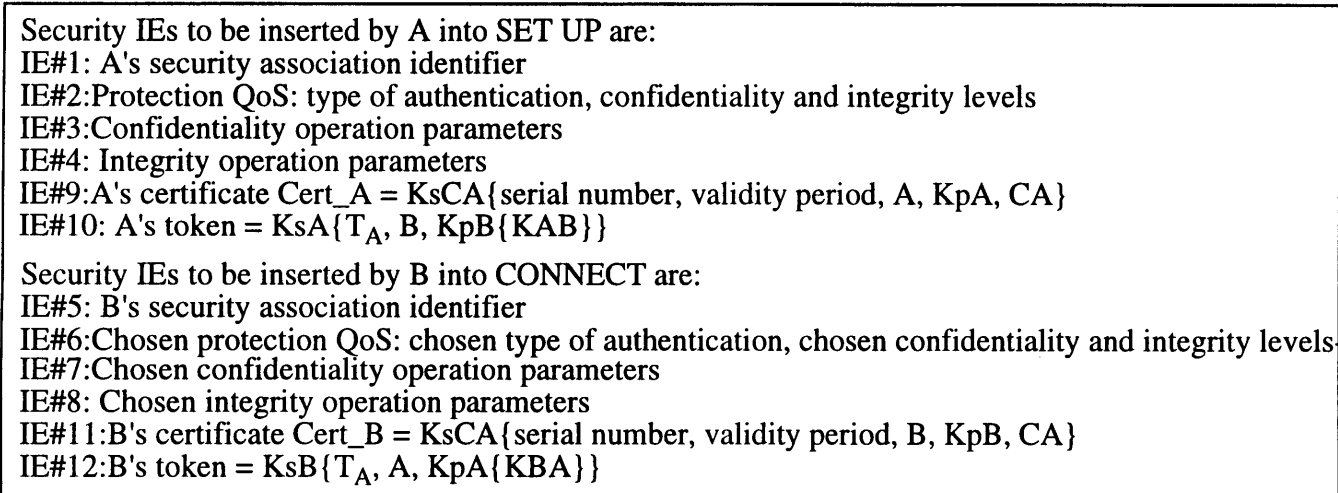

Figure 3 Deng's solution IEs.

As it states in Figure 2, the authentication IE is inaccurate and flawed. Firstly, the IEs ordering that should be enforced when generating the signature is not specified in case signaling messages include repeated IEs (of the same type). Secondly, it would be more efficient to distinguish end-to-end authentication IE from hop-by-hop authentication IE by assigning each a specific IE identifier instead of using the $E / E$ bit, since switches would no longer have to examin the Authentication IE fields up to the $E / E$ bit to know if authentication is to be performed. Thirdly, the authentication IE enables stations authentication only. Fourthly, there is one scenario that results in a malicious station, say $\mathrm{C}$, masquerading as $\mathrm{A}$. Assume that $\mathrm{A}$ authenticates to $\mathrm{B}$ with an authentication IE whose signature is not computed over B's address. C (a station eavesdropping the network or even B) may retrieve the authentication IE and reuse it to authenticate to another station, say $\mathrm{D}$ as $\mathrm{A}$. This assumes that the Sequence number is appropriate and $\mathrm{C}$ is quick enough so that the Timestamp remains valid.

On the other hand, the Version field makes this scheme flexible since it allows new authentication IEs to be defined and used simultaneousy on the same network.

\subsubsection{Deng et al.'s solution}

Deng defines twelve new IEs - six for each SET UP and CONNECT message - which allow entities $\mathrm{A}$ and $\mathrm{B}$ to authenticate mutually, exchange unidirectional session keys (2 IEs) and negotiate a security context (4 IEs).

Only confidentiality and integrity services to be applied on user data may be negotiated. The calling entity A proposes a set of security parameters to B within SET UP in four IEs (IEs \#1 to \#4 - see Figure 3). Parameters include its desired protection QoS and the lists of available confidentiality and integrity parameters. B replies with its chosen protection QoS and parameters in CONNECT (IEs \#5 to \#8). Then A accepts or refuses the connection depending on B's choices. Both A and B store the security context in the form of a security association identified respectively by A and B 's identifier (IEs \#1 and \#5).

Authentication and keys exchange are based on X509 two-way authentication protocol. The first X509 message within SET UP includes A's public key certificate (IE\#9) that allows $B$ to retrieve A's public key and a token $\mathrm{KsA}\left\{\mathrm{T}_{\mathrm{A}}, \mathrm{B}, \mathrm{KpB}\{\mathrm{KAB}\}\right\}$ (IE\#10) that allows $B$ to authenticate A and retrieve A's session key KAB. The X509 message returned by $B$ within CONNECT (IEs \#11 and \#12) is similar.

This scheme is rigid since the authentication protocol is imposed. Moreover using as many IEs as security parameters makes this scheme clear but not optimized in terms of modifications implied within UNI specifications (ATM Forum, 1994). 


\subsubsection{Chuang's solution}

Chuang developed its solution within the MSN-CMA architecture (Multi-Service Network Connection Management Architecture) which is based on servers supervising all ATM network's management and control operations. That is, when A needs to set up a connection, A sends its signaling message to the appropriate server over the ATM network by the means of Remote Procedure Call (RPC) mechanisms. The server then finds the appropriate end-to-end path, informs B of the request and sets switches' routing tables appropriately.

Contrary to the ATM Forum and the ITU-T, a connection set up requires the exchange of three signaling messages instead of two so that two-way and three-way authentication protocols may be chosen for that solution to authenticate and exchange session keys. Since the three-way protocols usually based on nonces are more reliable than two-way protocols based on timestamps, Chuang's solution appears more reliable than those of the previous sections.

The three-way handshake connection set up enables to negotiate, amongst others, confidentiality, integrity and keys exchange mechanisms to be used for securing user data exchanges. Also Chuang suggests that security parameters renegotiation be done through signaling exchanges.

\subsubsection{Negotiation through management information}

This scheme consists in injecting security information in-band within the user data channel by the means of OAM cells (cf. section 2).

\subsubsection{The ATM Forum's solution}

The ATM Forum uses such a scheme when security information requires synchronization with the user data cells stream, for instance to exchange new session keys during a connection.

The ATM Forum defines new OAM cells dedicated to security in order not to confuse them with ordinary OAM cells and suggests to encapsulate security messages into the 45-byte OAM cells' function-specific field. To protect security messages exchanges, protocols based on symetric or asymetric algorithms are specified.

\subsubsection{Negotiation through an auxiliary channel}

Auxiliary channel notion is used here when security information is exchanged in the form of user data cells.

\subsubsection{Stevenson et al.'s solution}

Stevenson suggests to negotiate session keys at connection set up within the first data exchanged.

Figure 4 depicts such a scheme in a proxies context. Proxies A and B are usually on line cryptographic units located at LAN boundaries whose function is to protect communications over the public network.

As shown in Figure 4, A sends a set up request to its proxy which in turn forwards it to proxy $\mathrm{B}$. Before completing the connection set up between $\mathrm{A}$ and $\mathrm{B}$, proxies $\mathrm{A}$ and $\mathrm{B}$ negotiate session keys by exchanging firstly their certificates and nonces and secondly their tokens which include unidirectional session keys and serve as authenticator. After keys negotiation, proxies complete the connection set up between $\mathrm{A}$ and $\mathrm{B}$ which are then allowed to exchange user data.

\subsubsection{The ATM Forum's solution}

This solution is used in conjonction with the solution of section 3.1.2.1 to negotiate se- 


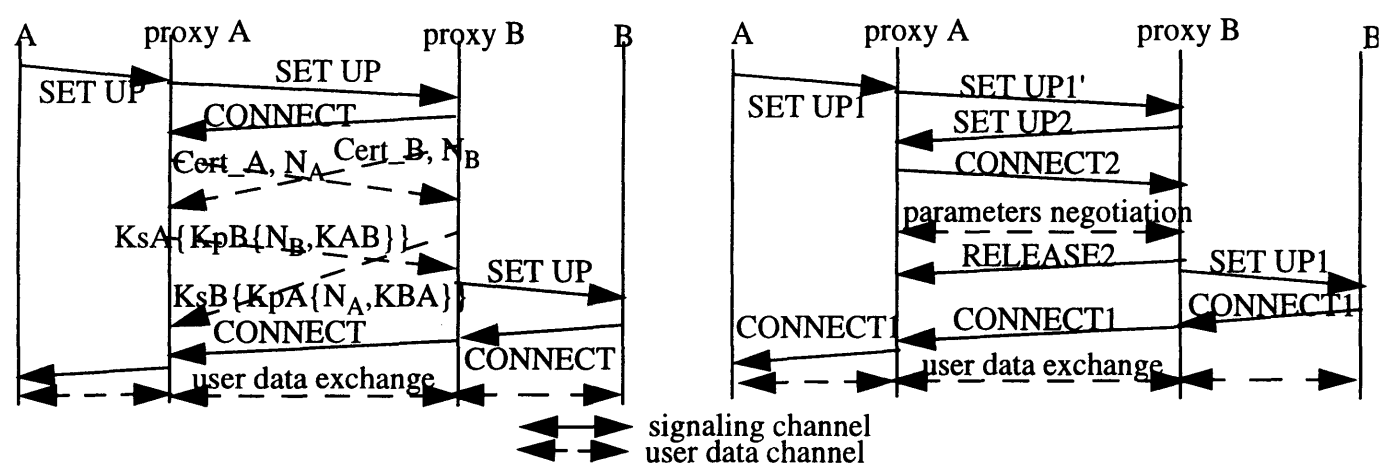

Figure 4 Session keys negotiation at connection set up via an auxiliary channel.

Stevenson et al.'s solution.

Figure 5 Parameters negotiation via the auxiliary channel.

The ATM Forum's solution.

curity parameters (security mechanisms). It may also be used to do mutual authentication during connection or at connection set up (in case a three-way authentication is required additionally to the two-way authentication realized within signaling messages in section 3.1.1.1).

This solution applies in a proxies context, however contrary to the Stevenson's solution, the auxiliary channel is a specific channel entirely dedicated to security parameters exchanges.

As shown in Figure 5, after receiving A's set up request (SET UP1), proxy A modifies the message into SET UP1' (by inserting security information such as a security services identifier) and forwards it to proxy B. Then proxy B detects the security services identifier and sets up a second connection with proxy A (SET UP2 and CONNECT2) over which all security parameters are negotiated. After the negotiation completion, proxies $\mathrm{A}$ and $\mathrm{B}$ release the second connection and complete the first connection between $\mathrm{A}$ and B.

\subsubsection{Comparisons of the solutions}

As shown in Table 3 (at the end of the paper), solutions may be compared in terms of modifications required within the ATM Forum's UNI 3.1 specifications (ATM Forum, 1994). Whereas Deng, respectively the ATM Forum, introduce twelve new IEs, respectively two new IEs and security OAM cells, Stevenson and Chuang require no UNI modifications since modifications are transparent for the ATM network. Stevenson modifies only the connection content and Chuang modifies processings within stations and servers (software).

All solutions include drawbacks listed hereafter.

The ATM Forum (section 3.1.1.1) and Deng's solutions expect intermediary public network equipments to transparently pass security information elements which may be used as covert channel (to pass data for free in case signaling is free). As a consequence, operators are reluctant to introduce new security IEs in signaling messages.

Both in-band and auxiliary channel schemes include flaws. The former is unsuitable for unidirectional communications and real-time applications. The latter is costly in terms of connections set ups (in ATM Forum 's implementation in section 3.1.3.2) or does not enable security parameters renegotiations during a connection (in Stevenson's implementation).

The environment of Chuang's solution is not compliant to ATM Forum and ITU-T's philosophy where management and control operations are distributed among network equipments so that his solution is not applicable to the current ATM standard environment. 


\subsection{Secure user data exchange}

User data exchanges may be protected with the security services negotiated in section 3.1.

Solutions differ in that user data encryption is performed:

- at the ATM layer (Stevenson, 1995), (Chuang, 1996), (ATM Forum, 1996)

- within the AAL layer (Deng, 1995)

\subsubsection{Confidentiality at the ATM layer}

This scheme consists in encrypting user data on the cell-by-cell basis. However encryption algorithms are more or less error-extension sensitive. That is, cell loss occurrence may cause for instance at most one 64-byte block loss when encrypted with DES ECB (Electronic Code Book) and much more data loss when encrypted with DES CBC (Cipher Block Chaining), since in the ECB mode, blocks of data are encrypted independently whereas in the $\mathrm{CBC}$ mode, an encrypted block of data is dependent on the plaintext block that generated it but also all the previous encrypted blocks. To avoid too many losses to occur, encrypting/decrypting devices need to be frequently resynchronized. Hereafter each solution's synchronization mechanism is specified.

\subsubsection{Stevenson et al.'s solution}

Stevenson defines cryptographic units (proxies of section 3.1.3.1) which encrypt ATM cells with one session key per connection. He studies the "key agility" problem, i.e. the problem of switching keys quickly enough so that ATM cells streams from various connections can be encrypted independently. He also solves the synchronization problem by injecting synchronization information in-band within OAM cells or user data cells.

\subsubsection{Chuang's solution}

Chuang defines a cryptonode device encrypting user data at the ATM layer with one session key per connection. Synchonization is performed at the AAL layer by injecting AAL5 PDU tokens including new keys numbers and initialization vectors (IV) that should be used for decrypting the next block of data. Therefore when a key or IV change is detected at the decryption device (two consecutive tokens contain different keys or IVs), an interrupt is generated to the ATM layer decryption process, so that cells decryption is halted and temporarily controled by the AAL layer for keys or IV update. In such a scheme, a problem may occur since synchronization is realized at a different layer than encryption. Indeed when a synchronization token is received mixed up with user data cells of the same connection, during the token processing at the AAL layer, many user data cells may be decrypted at the ATM layer with the old security context (old keys or IVs).

Additionally to synchronization, tokens support user data integrity/authentication by the means of a signature appended to tokens together with integrity keys and IV.

\subsubsection{The ATM Forum's solution}

The ATM Forum supports user data confidentiality and integrity (with two independent keys) and optionally reordering/replay detection. Confidentiality may be realized endto-end or switch-to-switch whereas integrity is only ensured end-to-end. Indeed, the confidentiality service is provided at the ATM layer but confidentiality at the AAL layer is also suggested for the end-to-end scenario.

The integrity and optional reordering/replay detection are supported at the AAL layer by the means of fields appended to the AAL SDU to protect: a signature computed over 
the AAL SDU, a timestamp and a sequence number. As mentioned in section 3.1.2.1, synchronization together with keys negotiation are realized in-band within OAM cells.

\subsubsection{Confidentiality within the AAL layer}

User data encryption is provided within the AAL layer prior to their segmentation into cells.

\subsubsection{Deng et al.'s solution}

Deng defines an additional layer called DPL (Data Protection Layer) which is placed between two AAL sublayers and supports confidentiality and integrity services and optional reordering. DPL SDUs are independently encrypted and signed with the keys negotiated at connection set up and the Initialization Vector (IV) specified within the DPL SDU's clear header. As a consequence no synchronization is needed.

During connection the DPL layer may update session keys by halting data transmission and sending repeatedly one DPL SDU containing a new session key (encrypted with its private key) until reception of a positive acknowledgement. This scheme appears unsuitable for unidirectional communications and real-time applications and is unsecure since no strong authentication protocols are used to exchange session keys.

\subsubsection{Solutions comparisons}

Solutions may be compared in terms of services supported. Stevenson's solution only supports the confidentiality service whereas all other solutions support confidentiality and integrity services.

We should note that the integrity service when offered is always placed at the AAL layer in order to avoid additional cells segmentations whereas the confidentiality service is placed at the ATM or AAL layer.

Encrypting user data at the ATM layer brings simplicity and performances improvements since encryption is realized on fixed-size cells and within hardware.

Benefits result also from encrypting user data at the AAL layer. Modifications within the ATM layered model are restricted to the AAL layer. Because of encryption realized on long-size block of data (up to 65535 bytes for DPL SDUs), no key agility problems raise. In Deng's solution, only session keys change requires synchronization with the user data flow and synchronization is easier to realize than in Chuang's solution since synchronization and encryption are done within the same layer like with ATM Forum and Stevenson's solutions.

However placing confidentiality at the AAL layer is less reliable than at the ATM layer since DPL PDUs and AAL PDUs' headers remain in clear.

It should be noticed that, contrary to ATM Forum and Chuang's solutions, the integrity and confidentiality services are not independent in Deng's solution since both of them use the same key. This results in algorithms choice restrictions and a weaker security level since in case the used key is broken, user data exchanged may be decrypted but also corrupted user data may be generated and signed.

\section{SOLUTION FOR FREQUENT COMMUNICATIONS (SFFC)}

We propose a solution which aims to protect communications between ATM stations but also ATM switches in case communications between ATM LANs need to be protected over the public network. This solution is especially suitable for ATM stations or LANs that frequently communicate and in particular, for companies needing to securely communicate with their remote subsidiaries. Later it is referred to as the Solution For Frequent Communications or SFFC. 
SFFC enables security services introduction within ATM stations or switches, thus defining ATM security stations or ATM security gateway switches. Such security devices are noted A and B respectively for the calling and called security devices.

Hereafter our solution is described in the same way as previous solutions, i.e. it is divided into two parts: security services negotiation and secure user data exchange.

\subsection{Security services negotiation}

The solution proposed is close to the ATM Forum's solution (section 3.1.1.1) since one security IE is introduced to provide both signaling messages source authentication and integrity protection.

As illustrated in Figure 6, the security IE includes fields similar to these of the ATM Forum's IE but is organized into security elements, each of which supports either security services or security services negotiation.

Hereafter security services offered by the IE are described:

- authentication and integrity of part of the signaling message.

Like with the ATM Forum's solution, the signature is computed over the security IE and part of the signaling message specified in the IEs list length and IEs list fields and the result is placed in the Digital Signature field. The repeated IEs' problem that raises in section 3.1.1.1 may be solved by introducing as many repeated IEs identifiers as repeated IEs to be signed into the IEs list. That is, if $\mathbf{n}$ repeated IEs' identifiers are introduced into IEs list, the signature is generated over the $\mathrm{n}$ first repeated IEs that appear within the signaling message. This is possible since repeated IEs' ordering is kept along the connection.

Contrary to the ATM Forum's solution, the signature algorithm to be used can be chosen by the interconnected ATM security devices. Indeed when A sends a security IE, A specifies a list of algorithms (with their respective information) ordered according to preferences within the field: List of algorithms to be used for signature. The first instance in the list is the algorithm code used to generate IE's signature. As such if B does not support that algorithm, B can choose another algorithm among the list and sends it back within a security IE. A will then use that algorithm to authenticate in other messages. A may also specify available algorithms that B should use for generating its signature in the security IE within the field List of algorithms to be used by the receiver if mutual authentication is required.

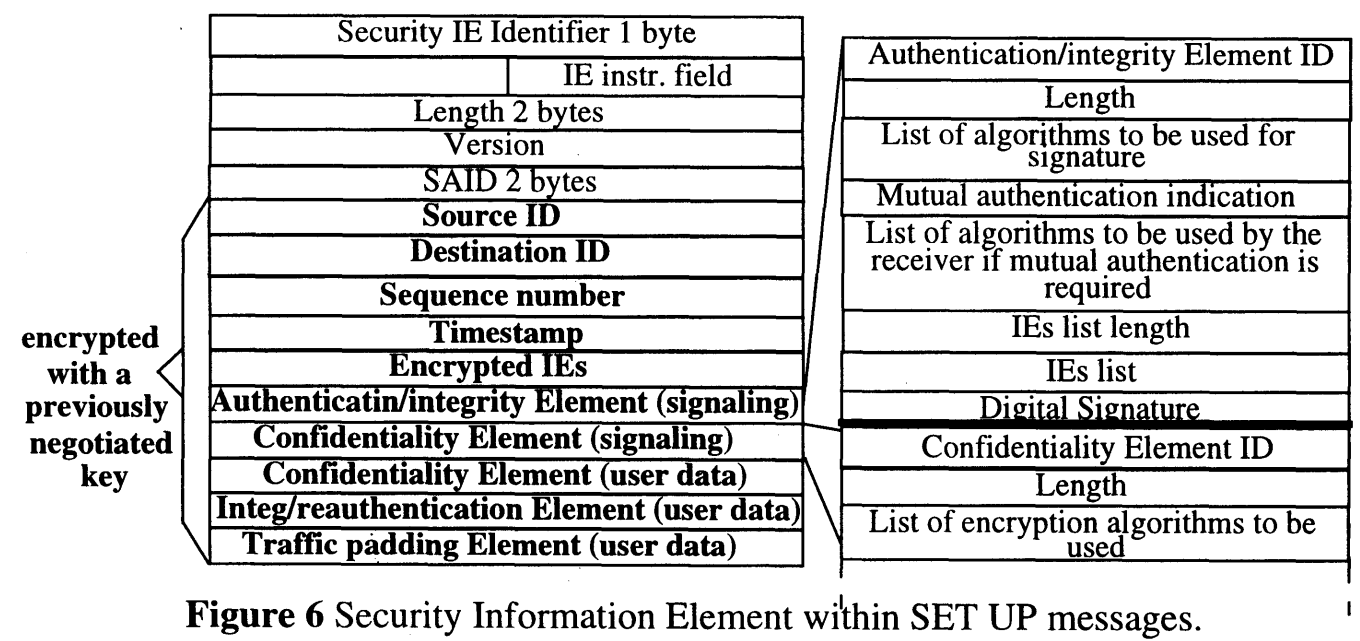


Contrary to the ATM Forum's solution, A may demand B to authenticate by the means of the field Mutual authentication indication. Also authentication based on both stations addresses and users is allowed since the fields - Source ID and Destination ID - may be used to carry specific users or user groups identifiers (alternative use of such fields is described below).

- confidentiality of part of the signaling message.

In the literature the signaling confidentiality service has never been studied since this assumes that a security context (i.e. session keys and encryption algorithms) has been previously negotiated. In the context where ATM security devices frequently communicate, we assume that a connection dedicated to security has already been set up between them to negotiate a list of session keys so that no keys exchanges are needed at connection set up. This security context is identified by a security association identifier ( $S A I D$ field) so that when receiving such a security IE, the decrypting device needs to retrieve the appropriate key within the SAID security context to decrypt the security IE.

The solution enables to encrypt part of security IE written in bold in Figure 6, i.e. security parameters included within security services elements but also ordinary signaling IEs that should not be analyzed by the public network and can be moved in the security IE in the encrypted IEs field to be encrypted.

Also this confidentiality service may be used to hide information within the security IE such as stations' addresses. This scheme appears suitable for companies wanting to keep their own addresses mappings secret while communicating over the network with their subsidiaries. This is realized by defining one global address common to all company's stations. Thus when setting up a connection with that company, the only clear address within the SET UP message (in the called entity number IE) is the global one and the real stations' addresses are encrypted within the Source ID and Destination ID fields of the security IE. This assumes that the security gateway switch B decrypts the security IE with the context identified by the SAID field before forwarding the set up request to the appropriate station.

- replay detection by the means of the Sequence number and Timestamp fields

- confidentiality (signaling and user data), integrity/reauthentication and traffic flow confidentiality ${ }^{*}$ services negotiation for the signaling and user plane

Four security elements allow A and B to negotiate mechanisms and session keys numbers to be used to provide such security services. A specifies a list of algorithms ordered according to preferences and B returns its choice within the next signaling message. Then choices are stored as the security context (identified by an SAID) to be applied on subsequent user data and signaling exchanges.

\subsection{Secure user data exchange}

We propose a solution close to Deng's solution (section 3.2.2.1) and the IEEE 802.10 model (IEEE 802.10A, 1989), (IEEE 802.10B, 1990) since all user data security services are introduced into one additional layer. This layer is placed above the AAL layer and called Secure Data Exchange layer (SDE) in reference to IEEE 802.10.

As depicted in Figure 7, the ATM Secure Data Exchange layer encapsulates user data $(S D E S D U)$ with one clear header and one encrypted header.

\footnotetext{
*. The traffic flow confidentiality service protects communications against traffic analysis by injecting spurious user data cells within user data flow
} 


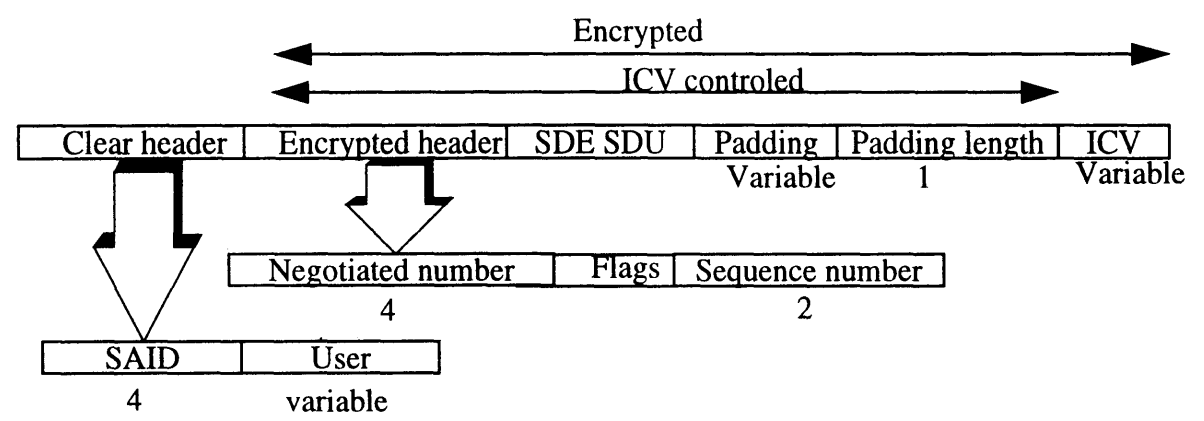

Figure 7 SDE PDU construction.

SDE offers the following security services:

- user data confidentiality. The SAID field identifies the security context (negotiated in section 4.1) used to encrypt user data (SDE SDU). Padding and Padding length fields are used to align data to be encrypted with a length suitable for the encryption algorithm.

- user data integrity/authentication. The Integrity Control Value (ICV) computed over the SDE PDU encrypted part may be used to both authenticate and check integrity. However in case the confidentiality service is offered, the ICV may be a simple Cyclic Redundancy Check (CRC) and the authentication service may be a secret number negotiated within the security IE and placed into the Negotiated number field.

- user data traffic flow confidentiality. Cells with no meaningful content may be injected into user data cells flow and recognized by the means of the Flag field.

- user data replay detection. User data unicity is ensured with the Sequence number field.

Like with Deng's solution, synchronization is realized on each SDE PDU by inserting into the User field synchronization information such as session keys numbers and initialization vectors to be used for encryption or ICV generation.

\section{IMPLEMENTATION}

We only implemented the security services negotiation part of our solution in SUN's ATM drivers environment (Paul, 1996). Since the ATM switch in our laboratory considers signaling messages with an additional security IE as error messages, our experimental network is limited to two SUN stations.

As depicted on Figure 8, we use TCP/IP stack on top of the ATM stack for testing purpose. Since SUN's ATM drivers use the concept of streams, our stack is divided into three streams:

- a first stream between TCP and IP

- a second stream one between IP and the ATM card's driver called SA. This stream is used to exchange user data and corresponds to the ATM model's user plane. The SA driver provides AAL5 services.

- a third stream between IP and SA including Q93B and SSCOP signaling protocols defined by ITU-T. This stream corresponds to the ATM model's control plane.

Signaling drivers include ONIP and Q93B. The latter checks signaling messages content to be compliant to the UNI specifications. We modified it, so it does not reject messages with a security IE. 


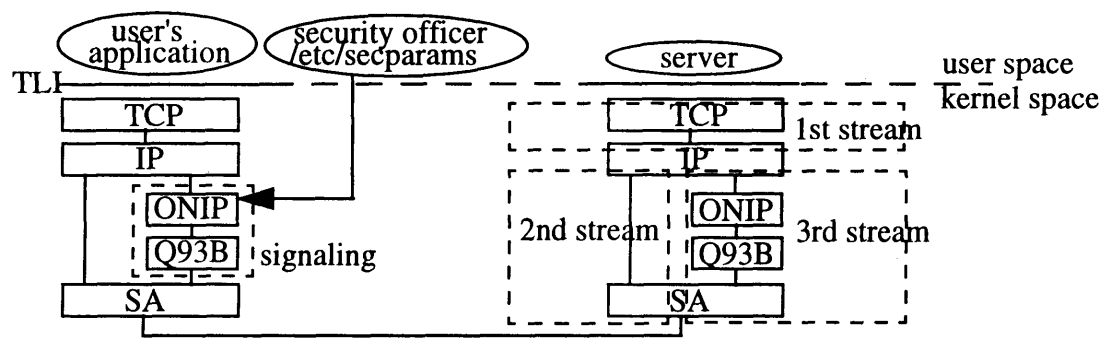

Figure 8 Our implementation.

Our implementation is based on another project "TCP Over Non-existent IP (ONIP)" realized at Télécom Bretagne which improves TCP performances by systematically causing an ATM connection set up for each TCP connection set up. This requires the following modifications: most of IP driver's functions are inhibited since they are realized in the AAL5 layer (e.g. cells segmentation/reassembly); an ONIP driver is created to monitor ATM connections and informs TCP of the identifiers of the connection being set up; TCP is modified in order to monitor ATM connections; the DNS server and its configuration files are modified to include ATM addresses.

Additionally to these modifications, we adapted cryptographic algorithms including DES, RSA, MD5 and keyedMD5 to the kernel space and created a cryptographic library. We modified the ONIP driver to build security IEs with the cryptographic library, i.e. compute fingerprints and encrypt part of the signaling message according to the security policy.

In order to retrieve the appropriate security policy when one TCP connection set up is requested, the TCP driver must send the appropriate SAID identifier together with its request to the ONIP driver. SAID can be buried in the user application thus allowing one security policy per application or computed at user login time. SAID is passed to the kernel by the means of a modified dynamically allocated TLI (Transport Level Interface) structure. Then ONIP driver can apply the appropriate security policy by consulting a kernel dynamically allocated structure which contains the SAID and the corresponding security policy. This structure can only be initialized or updated by the security officer with a root user program that reads a security policy file called /etc/secparams. After security services negotiation, ONIP driver has only to save the chosen security services and parameters with the user data connection identifiers in another structure.

One possibility to implement the SDE layer of section 4.2 is to place one additional driver CRYPT between IP and SA (along the second stream) which would provide user data security services. In order that CRYPT would retrieve the security policy negotiated at connection set up by ONIP, one solution would be that ONIP sends it to IP which would forward it to CRYPT.

\section{CONCLUSIONS}

In this paper, we present and compare solutions proposed by Stevenson et al., Deng et al., ATM Forum and Chuang to secure ATM networks. Thus, contrary to ATM Forum which meets both operators and users' security needs, Stevenson, Chuang and Deng focus on users' needs. That is, their philosophy is to ensure end-to-end communications security over an ATM public network considered as unsecure.

We describe also a new solution - the Solution For Frequent Communications (SFFC) which aims to secure communications between ATM stations or ATM LANs switches where stations or LANs need to communicate frequently. This solution is close to ATM 
Table 2 : Recapitulation of security services offered by ATM security solutions

\begin{tabular}{|c|c|c|c|c|c|}
\hline$a$ & $\begin{array}{l}\text { Stevens } \\
\text { on et al. }\end{array}$ & Deng et al. & Chuang & ATM Forum & $\begin{array}{l}\text { Télécom } \\
\text { Bretagne }\end{array}$ \\
\hline $\begin{array}{l}\text { security } \\
\text { services } \\
\text { established } \\
\text { through } \\
\text { signaling }\end{array}$ & & $\begin{array}{l}\text { A, KE, RD, } \\
\text { SN:I+C }\end{array}$ & $\begin{array}{l}\text { A, KE, RD, } \\
\text { SN:I+C+K } \\
\text { E }\end{array}$ & $\begin{array}{l}\text { A, I, AC, } \\
\text { KE, RD } \\
\text { SN:I+C+KE } \\
+\mathrm{A} \\
\\
\text { for all } \\
\text { signaling } \\
\text { messages }\end{array}$ & $\begin{array}{l}\mathrm{A}, \mathrm{I}, \mathrm{C}, \mathrm{RD}, \\
\mathrm{SN}: \mathrm{C}+\mathrm{I}+\mathrm{A}+\mathrm{P} \\
+\mathrm{RD}) \\
\\
\text { for all } \\
\text { signaling } \\
\text { messages }\end{array}$ \\
\hline $\begin{array}{l}\text { security } \\
\text { services } \\
\text { established } \\
\text { through } \\
\text { management } \\
\text { cells }\end{array}$ & & & & KE & \\
\hline $\begin{array}{l}\text { security } \\
\text { services } \\
\text { established } \\
\text { through the } \\
\text { auxiliary } \\
\text { channel }\end{array}$ & $\begin{array}{l}\text { EC, A, } \\
\text { RD }\end{array}$ & & & SN, A, KE & \\
\hline $\begin{array}{l}\text { user data } \\
\text { security } \\
\text { services }\end{array}$ & $\begin{array}{l}\text { C } \\
\text { within } \\
\text { ATM } \\
\text { S with } \\
\text { OAM } \\
\text { or user } \\
\text { data } \\
\text { cells }\end{array}$ & $\begin{array}{l}\mathrm{C}, \mathrm{I}, \text { and } \\
\text { (RD) } \\
\text { within } \\
\text { AAL } \\
\text { S done for } \\
\text { each DPL- } \\
\text { PDU } \\
\text { KE }\end{array}$ & $\begin{array}{l}\text { C within } \\
\text { ATM } \\
\text { I, A within } \\
\text { AAL } \\
\text { S within } \\
\text { AAL with } \\
\text { a cryptotag }\end{array}$ & $\begin{array}{l}\text { C rather } \\
\text { within } \\
\text { ATM } \\
\text { I, A and } \\
\text { (RD) } \\
\text { within } \\
\text { AAL } \\
\text { S with } \\
\text { OAM cells }\end{array}$ & $\begin{array}{l}(\mathrm{C}, \mathrm{I}, \mathrm{A}, \mathrm{RD}, \\
\mathrm{P}) \text { above } \\
\text { AAL layer } \\
\text { S on each } \\
\text { secure SDE } \\
\text { PDU }\end{array}$ \\
\hline
\end{tabular}

a. Notations used:

A Authentication

AC Access Control

KE Keys Exchange

C Confidentiality

P Padding

I Integrity

RD Replay Detection

(XX) Optional service XX

SN:XX Negociation of service XX

Forum and Deng's solutions, however as described in Table 2, it integrates additional security services. Amongst others, it ensures confidentiality of part of the signaling, thus allowing interconnected LANs to keep their addresses mappings secret. It allows to perform security services negotiation within signaling and, contrary to Chuang and Deng's 
Table 3 : ATM security solutions recapitulation

\begin{tabular}{|c|c|c|c|c|c|}
\hline & \begin{tabular}{|c|}
$\begin{array}{c}\text { Stevenson et } \\
\text { al. }\end{array}$ \\
\end{tabular} & Deng et al. & Chuang & ATM Forum & SFFC \\
\hline $\begin{array}{l}\text { security } \\
\text { parameters } \\
\text { renegotiation }\end{array}$ & no & \begin{tabular}{|l|} 
yes, keys \\
exchange \\
through DPL \\
PDUs
\end{tabular} & $\begin{array}{l}\text { yes, with } \\
\text { three } \\
\text { additional } \\
\text { signaling } \\
\text { messages } \\
\text { exchange }\end{array}$ & $\begin{array}{l}\text { yes, with } \\
\text { management } \\
\text { information }\end{array}$ & $\begin{array}{l}\text { yes, via a } \\
\text { secure } \\
\text { auxiliary } \\
\text { connection }\end{array}$ \\
\hline $\begin{array}{l}\text { authentication } \\
\text { and/or keys } \\
\text { negotiation } \\
\text { protocol }\end{array}$ & $\begin{array}{l}\text { protocols } \\
\text { based on } \\
\text { asymetric } \\
\text { algorithms }\end{array}$ & \begin{tabular}{|l|}
$\mathrm{X} 509$ two- \\
way protocol
\end{tabular} & $\begin{array}{c}\text { two-way or } \\
\text { three-way } \\
\text { protocols }\end{array}$ & $\begin{array}{l}\text { within the } \\
\text { signaling } \\
\text { flow: two- } \\
\text { way } \\
\text { protocols } \\
\text { within the } \\
\text { auxiliary } \\
\text { channel: } \\
\text { not defined }\end{array}$ & $\begin{array}{l}\text { two-way } \\
\text { protocols }\end{array}$ \\
\hline $\begin{array}{l}\text { modifications } \\
\text { required } \\
\text { within UNI }\end{array}$ & \begin{tabular}{|l|} 
none (unless \\
synchronizat \\
ion is \\
realized \\
through \\
security \\
OAM cells)
\end{tabular} & 12 new IEs & \begin{tabular}{|l|} 
none - \\
software \\
modification \\
s only
\end{tabular} & $\begin{array}{l}2 \text { new IEs and } \\
\text { new security } \\
\text { OAM cells }\end{array}$ & one new IE \\
\hline $\begin{array}{l}\text { distinction } \\
\text { between } \\
\text { confidentiality } \\
\text { and integrity } \\
\text { services }\end{array}$ & & $\begin{array}{l}\text { no, } \\
\text { mechanisms } \\
\text { are different } \\
\text { but keys are } \\
\text { similar }\end{array}$ & $\begin{array}{l}\text { yes, } \\
\text { mechanisms, } \\
\text { keys and } \\
\text { IVs are } \\
\text { different }\end{array}$ & yes & $\begin{array}{l}\text { yes, } \\
\text { mechanisms, } \\
\text { keys and } \\
\text { IVs are } \\
\text { different }\end{array}$ \\
\hline
\end{tabular}

solutions, that solution offers negotiated security parameters confidentiality. Moreover it enables both users and stations authentication. Additionally to the user data confidentiality and integrity services offered in Deng's solution, it offers user data reauthentication along the connection, replay detection and traffic padding.

\section{ACRONYMS}

AAL: ATM Adaptation Layer

IE: Information Element

IV: Initialization vector

OAM: Operation And Maintenance

QoS: Quality of Service
SAID: Secure Association Identifier

SDE: Secure Data Exchange

SDU: Service Data Unit

UNI: User Network Interface 


\section{REFERENCES}

ATM Forum (1994) ATM User-Network Interface Specification, version 3.1.

ATM Forum (1996) ATM Forum 95-1473R3, Phase I ATM security specification, June 1996.

Chuang S.C. (1996) Securing ATM networks. Third ACM conference on computer and communication security, New Delhi, India.

CCITT I.321 (1991) B-ISDN Protocol Reference Model and its applications.

Deng R.H., Gong L. and Lazar A.A. (1995) Securing data transfer in Asynchronous Transfer Mode networks, Proceedings of Globecom'95, pp 1198-1202, Singapore.

McDysan D.E. and Spohn D.L. (1994) ATM: Theory and application, McGraw-Hill series on Computer Communications.

IEEE 802.10A (1989) Standard for Interoperable LAN security (SILS) - Part A: The Model.

IEEE 802.10B (1990) Standard for Interoperable LAN Security (SILS) - Part B: Secure Data Exchange.

Paul O. (1996) Conception et implémentation d'un module de sécurité pour les réseaux ATM, Report ME-96001, Télécom Bretagne.

De Prycker M. (1991) Asynchronous Transfert Mode: Solution for broadband ISDN, Ellis Horwood, New York.

Stevenson D., Hillery N. and Byrd G. (1995) Secure Communications in ATM Networks, Communications of the ACM, Vol. 38, No. 2.

UIT-T X.800 (1991) Data communication networks ; open systems interconnection (OSI) ; security, structure and applications. Security architecture for open systems interconnection for CCITT applications.

\section{BIOGRAPHY}

Maryline LAURENT obtained an engineering degree in electronics from the French graduate School ENSERB in 1993 and an advanced degree in Telecommunication Network Management from the Franco Polish School of New Information and Communication technology (EFP). Now she is a PhD student within the Networks and Multi-media Services Department (RSM) of Telecom Bretagne (a French Graduate School of Telecommunications Engineering), Rennes, France, since December 1994. She works on networks security, mainly in the ATM field, where her job consists in securing communications over ATM networks. 